\title{
Avaliação da auto-percepção de competência: adaptação da PSPCSA numa população portuguesa
}

\author{
Maria Adelina Barbosa Ducharne ${ }^{1}$
}

\begin{abstract}
Resumo
A Pictorial Scale of Perceived Competence and Social Acceptance for Young Children, é talvez dos instrumentos que mais têm sido utilizados na investigação sobre a auto-percepção da criança de idade pré-escolar, bem como pelos psicólogos que, na sua prática profissional, lidam com este tipo de questão. O presente estudo teve como objectivo proceder à adaptação da PSPCSA às características de uma amostra portuguesa de crianças de idade pré-escolar. Serão descritos os participantes, o processo de tradução/adaptação da escala, o procedimento e os resultados, bem como serão discutidas as implicações dos resultados obtidos na utilização desta escala com crianças portuguesas de idade pré-escolar. A versão portuguesa da PSPCSA apresenta algumas diferenças relativamente à escala original americana, nomeadamente no que diz respeito à estrutura factorial. As qualidades psicométricas da EAPCASC portuguesa foram consideradas muito satisfatórias, e as mudanças incluídas na sequência do processo de adaptação revelaram-se indispensáveis.

Palavras-Chave: Desenvolvimento do self; Crianças de idade pré-escolar; Avaliação da auto-percepção de competência; EAPCASC.
\end{abstract}

\section{Evaluation of self-perceived competence: A Portuguese adaptation of the PSPCSA}

\begin{abstract}
The Pictorial Scale of Perceived Competence and Social Acceptance for Young Children has been used widely by researchers and practicians interested in young children' self-perception. Considering both the advantages of a reliable and valid instrument of young children self-perception assessment, and that a sound use of this scale with children different from the original sample requires a diligent work of adaptation, the present study was designed to adapt the PSPCSA to the cultural features of a Portuguese population. Participants, instrument development, procedures and results will be presented as well as the discussion of the implications of the results obtained in the utilization of this instrument with Portuguese preschool children. The Portuguese version of the PSPCSA shows some differences from the original American, especially in what concerns factorial structure: the pattern found being quite different. The psychometrics qualities of the Portuguese version are considered very acceptable and the included changes appear indispensable.

Keywords: Self-development; Preschool children; Evaluation of self-perceived competence; Portuguese adaptation; PSPCSA.
\end{abstract}

A Pictorial Scale of Perceived Competence and Social Acceptance for Young Children (PSPCSA; Harter \& Pike, 1983) é talvez dos instrumentos mais utilizados, quer por investigadores, quer por clínicos interessados na autopercepção de competência de crianças de idade préescolar. De facto, desde a sua publicação do início da década de 80 tem sido utilizada com objectivos clínicos, educativos e de investigação, não apenas nos Estados Unidos, mas em múltiplos e diferentes contextos culturais.

A PSPCSA é um instrumento que pretende avaliar a auto-percepção de competência e aceitação social de crianças entre os quatro e os sete anos. Concretamente, esta escala prevê duas formas: uma, a preschool kindergarten, é susceptível de ser utilizada com crianças de quatro ou cinco anos; a segunda, a first and second grades, reporta-se a crianças de seis ou sete anos de idade. Cada uma destas formas é constituída por um total de 24 itens, repartidos por quatro sub-escalas, com seis itens cada. As quatro sub-escalas referem-se, respectivamente, à percepção de competência cognitiva, à percepção de competência física, à percepção de aceitação pelos pares e à percepção de aceitação materna.

Trata-se de uma escala pictórica de aplicação individual, ou seja, os itens são apresentados à criança a partir de uma imagem que serve de suporte ao texto, lido pelo experimentador. Tal como referem os autores, "o formato pictórico desperta o interesse e mantém a atenção da criança, é facilmente compreendido e conduz a respostas mais significativas" (Harter \& Pike, 1984, p. 1970). Por sua vez, todos os itens estão redigidos sob um formato especial, com objectivo de reduzir ao máximo as respostas socialmente desejáveis, isto é, os

\footnotetext{
${ }^{1}$ Endereço para correspondência:

Rua do Campo Alegre, 1055 - 4169-004 - Porto - Portugal

E-mail: abarbosa@fpce.up.pt
} 
itens são apresentados sob forma dicotómica, permitindo, no entanto, uma resposta numa escala de quatro pontos. De facto, em cada situação a criança é confrontada com duas outras do mesmo sexo da criança-alvo; uma das quais é descrita como competente na realização de determinada tarefa, ou socialmente aceite, enquanto a segunda é apresentada como menos competente ou menos aceite. Perante esta duas outras, a criança-alvo deve indicar aquela que considera mais parecida consigo mesma. Uma vez a escolha feita, a criança é novamente confrontada com uma alternativa, na qual deve posicionar a percepção que tem da sua competência ou da sua aceitação pelos outros (os pares ou a mãe). A nota obtida para cada item varia entre um mínimo de um ponto e um máximo de quatro pontos.

Uma vez que a escala comporta duas formas, preschool kindergarten e first and second grades, a análise factorial foi operada separadamente para duas amostras de crianças, respectivamente, de 145 e 104 crianças. No entanto, em ambas as análises emergiram dois factores: um factor de competência, no qual saturam os itens das duas sub-escalas de percepção de competência, cognitiva e física, e um factor de aceitação, no qual saturam os itens das duas sub-escalas de percepção de aceitação, pelos pares e materna. Tal dado é interpretado pelos autores da escala como uma incapacidade das crianças neste nível desenvolvimental, de proceder a discriminações entre domínios específicos de percepção de competência e de percepção de aceitação social.

$\mathrm{O}$ alfa de Cronbach foi calculado como índice de consistência interna, quer para cada uma das quatro sub-escalas, quer para as duas escalas (de percepção de competência e de percepção de aceitação), quer ainda para a escala total. Os resultados obtidos para cada sub-escala variaram entre valores baixos a muito satisfatórios, na medida em que oscilaram entre 0,53 e 0,83 . No que se refere às duas escalas de percepção de competência e de percepção de aceitação, os índices de consistência obtidos foram muito satisfatórios, variando entre 0,76 e 0,87 . Finalmente, para a escala completa são apresentados como índices de consistência interna 0,88 e 0,87 para a forma pré-escolar e para a forma escolar, respectivamente.

Tendo em conta, por um lado, as vantagens de dispor de um instrumento fidedigno e validado de avaliação da auto-percepção da criança e, por outro, o reconhecimento de que a utilização de um instrumento de avaliação psicológica junto de uma população com características diferentes da amostra original implica num aturado processo da adaptação, o presente estudo teve como objectivo proceder à adaptação da PSPCSA às características de uma amostra portuguesa de crianças de idade pré-escolar.

\section{Método}

\section{Participantes}

A amostra deste estudo é uma amostra de conveniência, constituída por 128 crianças de 5 anos. Esta amostra foi escolhida a partir da população de crianças de cinco anos que frequentava o jardim infantil, em equipamentos públicos ou privados, na cidade do Porto.

Os critérios que presidiram à selecção das crianças que fariam parte da amostra foram definidos em função dos objectivos de um estudo mais amplo que pretendia identificar os factores preditores da autopercepção de competência em crianças de idade escolar (Ducharne, 2000). Assim, de entre as crianças que frequentavam um dos 27 jardins-de-infância na cidade do Porto, foram escolhidas aquelas que tinham cinco anos de idade (entre 5;0 e 5;11 anos), eram filhos primogénitos, pertenciam a famílias bi-parentais, e podiam ser incluídas num de três grupos (alto, médio ou baixo), quanto ao nível sócio-cultural da família definido a partir dos anos de escolaridade da mãe e do pai. Procurou-se obter o mesmo número de meninas e rapazes em cada um dos três níveis sócio-culturais considerados. Foram excluídas as crianças apontadas pelas educadoras como apresentando atrasos importantes de desenvolvimento.

O nível sócio-cultural da família foi definido a partir do número de anos de estudo completados pelo pai e pela mãe, sendo que a pertença a "nível alto" implica que mãe e pai sejam licenciados, ou pelo menos um deles o seja, e o outro tenha 12 ou mais anos de escolaridade; "nível médio" implica que mãe e pai tenham 9 ou mais anos de escolaridade e nenhum seja licenciado; "nível baixo implica que mãe e pai tenham menos de 8 anos de escolaridade. Os termos em que estes critérios foram definidos implicam uma certa homogeneidade intracasal a nível das habilitações literárias, o que de facto veio a acontecer e é demonstrado pela correlação (coeficiente de Pearson) elevada encontrada entre o número de anos de estudo completados pela mãe e o número de anos de estudo completados pelo pai $(r=0,79, p<0,0001)$.

Tabela 1 - Distribuição da amostra em função do nível sócio-cultural e do sexo das crianças $(\mathrm{N}=128)$

\begin{tabular}{lcccc}
\hline & \multicolumn{4}{c}{ Meio sócio-cultural da família } \\
\cline { 2 - 5 } Sexo & Alto & Médio & Baixo & Total \\
\hline Feminino & $23(18,0)$ & $20(15,6)$ & $20(15,6)$ & $63(49,2)$ \\
Masculino & $21(16,4)$ & $22(17,2)$ & $22(17,2)$ & $65(50,8)$ \\
Total & $44(34,4)$ & $42(32,8)$ & $42(32,8)$ & $128(100)$ \\
\hline
\end{tabular}


A Tabela 1 apresenta a distribuição da amostra por meio sócio-cultural da família e sexo da criança. Entre parêntesis apresentam-se as percentagens de cada célula relativamente ao total. Como se pode apreciar na leitura da Tabela 1, não há associação entre sexo e nível sócio-cultural, $\left(\chi^{2}(2)=0,25, \mathrm{n}\right.$. s.), o que deriva, obviamente, de estas variáveis terem sido usadas como critério de selecção da amostra. Na realidade, a amostra foi escolhida de modo a obter-se sensivelmente o mesmo número de crianças em cada uma das seis células determinadas pelo cruzamento destas duas variáveis.

\section{Tradução/Adaptação da escala}

A Escala de Auto-Percepção de Competência e Aceitação Social para Crianças, em imagens, versão para crianças de idade pré-primária, (EAPCASC), constitui uma versão traduzida e adaptada da PSPCSA, forma preschool kindergarten (Harter \& Pike, 1983). A EAPCASC é o resultado de um aturado trabalho de tradução e adaptação da versão americana original, sendo constituída por 31 itens distribuídos por quatro sub-escalas.

A adaptação à realidade cultural portuguesa da escala original americana envolveu várias etapas, desde a tradução até à análise das imagens que servem de suporte, culminando na condução de um estudo-piloto cujos resultados determinaram a versão final do instrumento. Num primeiro momento, as duas versões da PSPCSA (pré-escolar e de primeiro e segundo graus) foram traduzidas e revistas por peritos familiarizados quer com a linguagem infantil quer com os constructos psicológicos envolvidos. Adicionalmente, procedeu-se a entrevistas com crianças da idade-alvo com objectivo de identificar outras competências ou situações sociais não incluídas na escala e susceptíveis de contribuir para o seu sentido de competência pessoal e aceitação social. Finalmente, algumas das imagens originais foram alteradas de modo a ser melhor compreendidas e outras foram acrescentadas, correspondendo a novos itens.

A versão assim obtida era constituída por 40 itens (dos quais 24 correspondiam à versão pré-escolar da PSPCSA, 10 pertenciam à versão primeiro e segundo graus da mesma escala e 6 eram itens novos) e foi submetida a um estudo-piloto, com uma amostra de 63 crianças (32 meninas e 31 rapazes), com idades compreendidas entre 60 e 71 meses ( $M=66$ meses, $\mathrm{DP}=3,6$ ), oriundas de famílias de diferente níveis sócioculturais e que frequentavam um jardim-de-infância na cidade do Porto. Este estudo-piloto evidenciou a boa aceitação por parte das crianças a este tipo de prova.
Os resultados do estudo-piloto conduziram à versão final do instrumento constituída por 31 itens, dos quais 23 coincidem com a escala original americana (foi excluído um item, pertencente à sub-escala de aceitação materna e relativo à frequência com que a mãe cozinha pratos que a criança gosta, dada a sua inadequação ao contexto cultural português) e os oito restantes são aqueles que demonstraram melhores qualidades psicométricas no estudo-piloto conduzido.

\section{Procedimento}

A EAPCASC foi administrada individualmente às 128 crianças da amostra por um de seis colaboradores de investigação especialmente treinados para o efeito. Dado que a escala compreende duas versões, uma feminina (em que nos desenhos surge uma figura de criança do sexo feminino) e outra masculina (a criança no desenho é de sexo masculino), foi utilizada com cada criança a versão adequada ao sexo. A administração da escala decorreu numa sala familiar à criança no seu jardim-de-infância e num ambiente agradável.

\section{Resultados}

Os dados foram analisados tendo em vista submeter a EAPCASC portuguesa aos procedimentos correntes de apreciação das suas qualidades psicométricas, nomeadamente a nível da sensibilidade dos itens, da sua estrutura factorial, bem como da sua fidelidade, através do índice de consistência interna; e da sua validade convergente discriminante, através do coeficiente de correlação de cada item com a sub-escala respectiva e com a escala completa. Apresentaremos de seguida os resultados destas análises, na sequência das quais se procederá à leitura descritiva dos dados obtidos com a escala. Embora se trate de medidas ordinais, seguindo a recomendação feita em várias obras da especialidade (Fife-Schaw, 1995; Miller, 1984; Reckase, 1990; Ribeiro, 1999), optou-se por recorrer à estatística paramétrica na medida em que é privilegiada a congruência entre os procedimentos estatísticos usados ao longo do estudo.

\section{Sensibilidade dos itens}

Tendo em vista apreciar a sensibilidade dos itens que fazem parte da EAPCASC portuguesa, procedeu-se à análise da distribuição obtida para cada item. A Tabela 2 resume esta análise, indicando a variação obtida para cada item, a respectiva média e desvio-padrão, bem como os valores de curtose e assimetria obtidos. 
Tabela 2 - Sensibilidade dos itens da EAPCASC: variação, média e desvio-padrão, bem como valores de curtose e assimetria obtidos, para cada item $(\mathrm{N}=128)$

\begin{tabular}{|c|c|c|c|c|c|}
\hline Item & Variação & Média & D-P & Curtose & Assimetria \\
\hline 1 - Sabe fazer puzzles & $1-4$ & 3,38 & 0,99 & 0,51 & $-1,36$ \\
\hline 2 - Tem amigos & $1-4$ & 3,17 & 1,06 & $-0,88$ & $-0,80$ \\
\hline 3 - Anda de balouço & $1-4$ & 3,55 & 0,84 & 1,41 & $-1,65$ \\
\hline 4 - Mãe bem-disposta & $1-4$ & 3,20 & 0,73 & $-0,60$ & $-0,45$ \\
\hline 5 -Educadora elogia & $2-4$ & 3,33 & 0,67 & $-0,73$ & $-0,49$ \\
\hline 6 - Convidado ir dormir & $1-4$ & 2,35 & 1,08 & $-1,32$ & 0,05 \\
\hline 7 - Trepa & $1-4$ & 3,14 & 1,02 & $-0,46$ & $-0,88$ \\
\hline 8 - Mãe leva passear & $2-4$ & 3,20 & 0,78 & $-1,25$ & $-0,37$ \\
\hline 9 - Conhece cores & $2-4$ & 3,78 & 0,50 & 4,43 & $-2,27$ \\
\hline 10 - Amigos p/jogar & $1-4$ & 3,27 & 0,94 & $-0,23$ & $-0,98$ \\
\hline 11 - Aperta sapatos & $1-4$ & 2,99 & 1,23 & $-1,25$ & $-0,66$ \\
\hline 13 - Sabe contar & $1-4$ & 3,46 & 0,79 & 0,34 & $-1,22$ \\
\hline 14 - Amigos p/brincar & $1-4$ & 3,35 & 0,91 & 0,11 & $-1,14$ \\
\hline 15 - Consegue saltar & $1-4$ & 3,59 & 0,70 & 1,44 & $-1,54$ \\
\hline 16 - Mãe lê histórias & $1-4$ & 2,80 & 1,05 & $-0,90$ & $-0,51$ \\
\hline 17 - Conhece inicial & $1-4$ & 3,66 & 0,69 & 3,54 & $-2,07$ \\
\hline 18 - Chamado p/brincar & $1-4$ & 3,10 & 0,87 & $-0,39$ & $-0,64$ \\
\hline 19 - Corre & $1-4$ & 3,69 & 0,72 & 5,29 & $-2,43$ \\
\hline 20 - Mãe brinca & $1-4$ & 2,60 & 1,05 & $-1,17$ & $-0,11$ \\
\hline 21 - Conhece letras & $1-4$ & 3,11 & 0,94 & $-0,62$ & $-0,68$ \\
\hline 22 - Convidado ir lanchar & $1-4$ & 2,90 & 0,96 & $-0,53$ & $-0,60$ \\
\hline 23 - Salta pé coxinho & $1-4$ & 3,38 & 0,90 & 0,65 & $-1,30$ \\
\hline 24 - Mãe conversa & $1-4$ & 3,28 & 0,85 & 0,10 & $-0,97$ \\
\hline 25 - Trabalhos J. I. & $1-4$ & 3,52 & 0,66 & 1,02 & $-1,24$ \\
\hline 26 - Amigos emprestam & $1-4$ & 3,33 & 0,87 & 0,67 & $-1,21$ \\
\hline 27 - Joga à bola & $1-4$ & 3,46 & 0,82 & 1,38 & $-1,47$ \\
\hline 28 - Mãe deixa ir lanchar & $1-4$ & 3,05 & 1,01 & $-0,48$ & $-0,79$ \\
\hline 29 - Sabe escrever nome & $1-4$ & 3,48 & 0,88 & 1,81 & $-1,66$ \\
\hline 30 - Outros sentam junto & $1-4$ & 3,32 & 0,89 & 0,19 & $-1,09$ \\
\hline 31 - É forte & $1-4$ & 3,36 & 0,93 & $-0,02$ & $-1,14$ \\
\hline 32 - Mãe deixa dormir fora & $1-4$ & 2,63 & 1,18 & $-1,44$ & $-0,27$ \\
\hline
\end{tabular}

Da leitura da Tabela 2, pode apreciar-se a distribuição obtida para os vários itens que constituem a EAPCASC. Assim, a quase totalidade dos itens apresentou uma variação entre o mínimo e o máximo possíveis (1-4), excepto os itens 5, 8 e 9. Verificou-se também que o item que apresenta maior média é o item $9(\mathrm{M}=3,87)$, sendo também aquele cujo desvio-padrão é menor $(\mathrm{DP}=0,50)$; o item que apresenta média mais baixa é o item $6(\mathrm{M}=2,35)$, e aquele cujo desvio-padrão é maior é o item 11 ( $\mathrm{DP}=1,23)$. Note-se que o item no 12 não existe. De facto, na versão original americana este era o item que foi suprimido por não se adequar à população portuguesa, mas optamos por manter a mesma numeração dos itens comuns às duas escalas. No que diz respeito às medidas de curtose e de assimetria, verifica-se que apenas dois itens apresentam valores indicativos de uma distribuição muito afastada da distribuição normal. São os itens 9 e 19 nos quais, de facto, se verifica uma concentração dos resultados nos valores mais elevados da escala. Em suma, pode considerar-se que os 31 itens que constituem a
EAPCASC portuguesa são, de modo geral, satisfatórios.

\section{Estrutura factorial}

A análise da estrutura factorial apresentada por esta escala obedeceu a critérios não meramente estatísticos, mas decorrentes de considerações teóricas. Assim, num primeiro momento, verificou-se a adequação da matriz de correlações à aplicação de uma análise de componentes principais, tal como é proposto por Norusis (1993). Foram considerados três indicadores. O primeiro indicador é dado pelo teste de esfericidade de Bartlett, o qual é utilizado para verificar a hipótese de a matriz de correlações ser uma matriz idêntica, ou seja, se todos os valores em diagonal são 1 e todos os outros são 0 . Os resultados desta análise permitem-nos concluir que se trata de dados provenientes de uma população multivariada normal $\left(\chi^{2}=1281,95, p<0,0001\right)$.

$\mathrm{O}$ segundo indicador da adequação da matriz de correlações à aplicação de uma análise de componentes principais é o coeficiente de correlação parcial. Se as 
variáveis partilharem factores comuns, os coeficientes de correlação parcial entre os pares de variáveis devem ser pequenos quando os efeitos lineares das outras variáveis forem eliminados. As correlações parciais são então estimativas das correlações entre os factores únicos e devem estar próximas de zero, quando os pressupostos da análise factorial são cumpridos. $\mathrm{O}$ índice de adequação de Kaiser-Meyer-Olkin compara as magnitudes dos coeficientes de correlação observados com as magnitudes dos coeficientes de correlação parcial. Quanto mais elevado for este índice, mais adequada é a realização da análise factorial. O índice obtido foi de 0,77 , o que é considerado bom.

Finalmente, o terceiro critério da adequação da matriz de correlações à aplicação de uma análise de componentes principais é o valor da comunalidade de cada item. A comunalidade de um item consiste no somatório das variâncias explicadas pelos diversos factores; quanto mais elevada fôr, mais a variância do item é explicada pelos factores comuns. Os valores da comunalidade $\left(\mathrm{h}^{2}\right)$ dos itens são apresentados na Tabela 3.

Num segundo momento (e uma vez assegurada a adequação de se proceder a uma análise de componentes principais, a partir de critérios estatísticos), optou-se por limitar a quatro o número de factores a se extrair, dado ser este o número de dimensões conceptualmente consideradas na construção da escala. Em suma, procedeu-se a uma análise de componentes principais, com rotação ortogonal (procedimento varimax), com limite de quatro factores. Estes quatro factores explicam, na sua totalidade, $41 \%$ da variância. $O$ primeiro factor destaca-se dos restantes, explicando 22,3\% da variância, enquanto os outros três explicam respectivamente $7,3 \%$, $6,3 \%$ e 5,1\%. A Tabela 3 apresenta os quatro factores com respectivos valores próprios (eigenvalues) e percentagem de variância explicada, as saturações factoriais dos 31 itens correspondentes, bem como a sua comunalidade.

Tabela 3 - EAPCASC: Saturações factoriais após rotação ortogonal e comunalidade $\left(\mathrm{h}^{2}\right)$ dos itens. Valores próprios (eigenvalues) e percentagem da variância explicada

\begin{tabular}{|c|c|c|c|c|c|}
\hline Itens & $h^{2}$ & $\overline{F 1}$ & $\mathrm{~F} 2$ & F3 & F4 \\
\hline 16 - Mãe lê histórias & 0,558 & 0,730 & & & \\
\hline 22 - Convidado ir lanchar fora & 0,523 & 0,680 & & & \\
\hline 28 - Mãe deixa ir lanchar & 0,497 & 0,673 & & & \\
\hline 24 - Mãe conversa & 0,523 & 0,668 & & & \\
\hline 32 - Mãe deixa ir dormir fora & 0,413 & 0,547 & & & \\
\hline 20 - Mãe brinca & 0,472 & 0,544 & & & \\
\hline 8 - Mãe leva a passear & 0,313 & 0,406 & & & \\
\hline 6 - Convidado ir dormir & 0,371 & 0,403 & & & \\
\hline 14 - Amigos p/ brincar & 0,682 & & 0,813 & & \\
\hline 10 - Amigos p/ jogar & 0,603 & & 0,739 & & \\
\hline 2 - Tem amigos & 0,518 & & 0,672 & & \\
\hline 30 - Outros sentam junto & 0,577 & & 0,655 & & \\
\hline 26 - Amigos emprestam brinquedos & & 0,431 & & 0,517 & \\
\hline 18 - Chamado p/ brincar & 0,428 & & 0,439 & & \\
\hline 15 - Consegue saltar & 0,479 & & & 0,609 & \\
\hline 23 - Salta a pé coxinho & 0,439 & & & 0,598 & \\
\hline 7 - Trepa & 0,441 & & & 0,597 & \\
\hline 19 - Corre & 0,301 & & & 0,537 & \\
\hline 31 - É forte & 0,352 & & & 0,519 & \\
\hline 1 - Sabe fazer puzzles & 0,326 & & & 0,442 & \\
\hline 3 - Anda de balouço & 0,289 & & & 0,440 & \\
\hline 27 - Joga à bola & 0,309 & & & 0,439 & \\
\hline 17 - Conhece inicial do nome & 0,471 & & & & 0,646 \\
\hline 5 - Educadora elogia & 0,412 & & & & 0,576 \\
\hline 29 - Sabe escrever o nome & 0,326 & & & & 0,514 \\
\hline 25 - Trabalhos J. I. & 0,407 & & & & 0,473 \\
\hline 4 - Mãe bem-disposta & 0,328 & & & & 0,464 \\
\hline 11 - Aperta sapatos & 0,206 & & & & 0,440 \\
\hline 13 - Sabe contar & 0,269 & & & & 0,362 \\
\hline 9 - Conhece cores & 0,181 & & & & 0,327 \\
\hline 21 - Conhece letras & 0,272 & & & & 0,238 \\
\hline Valores próprios (eigenvalues) & & 6,93 & 2,25 & 1,96 & 1,58 \\
\hline \% variância & & 22,3 & 7,3 & 6,3 & 5,1 \\
\hline
\end{tabular}


A análise do conteúdo dos itens que saturam em cada factor permite a sua interpretação nos seguintes termos:

- Oito itens saturam no factor 1 , quatro dos quais se referem a actividades que a mãe realiza com a criança (ler histórias, conversar, brincar e levar a passear), enquanto os quatro restantes implicam um certo afastamento da mãe, ou seja, a criança é convidada e a mãe autoriza a sua ida a lanchar ou dormir a casa de pessoas amigas. Embora a intenção inicial dos dois itens que se referem a convites fosse obter uma apreciação do sentimento que a criança tem de quanto a sua presença é requerida pelos pares para partilhar quer uma refeição, quer o período da noite, o que na realidade parece ocorrer é que as crianças não fazem grande distinção entre o convite para ir e a ida de facto, assimilando as duas situações. Neste sentido, parece-nos que este primeiro factor traduz a percepşão da qualidade da relaşão com a mãe, quer nas actividades que implicam proximidade, quer na capacidade de se distanciar daquela;

- Seis itens, que se referem ao sentimento da criança de que tem amigos que partilham com ela actividades e brinquedos e à percepção que tem de quanto a sua companhia é procurada pelos outros, saturam no factor 2. Neste sentido, parece-nos que este segundo factor traduz a percepcão da qualidade da relação com os pares;

- Oito itens que se referem à percepção que a criança tem da competência com que realiza uma série de actividades habituais no jardim infantil saturam no factor 3. Exceptuando o item 1, que se refere à realização de puzzles, todos os restantes implicam actividades de motricidade grossa. No entanto, o que nos parece haver de comum entre as diversas actividades e que poderá estar subjacente à compreensão que a criança tem dos oito itens é que todos eles se referem a acções que são naturalmente aprendidas pelas crianças, no contexto do jardim infantil. Neste sentido, parece-nos que este terceiro factor traduz a percepscão de competência na realização de actividades naturalmente aprendidas. De facto, embora a escala original estabeleça a distinção entre actividades motóricas e cognitivas, não nos parece que, do ponto de vista das crianças, tal dicotomia faça sentido;

- Finalmente, nove itens saturam no factor 4. Destes nove itens, sete referem-se à percepção que a criança tem da competência com que realiza uma série de actividades habituais no jardim infantil, como por exemplo identificar letras (entre as quais a inicial do nome), saber escrever o nome próprio, saber contar, conhecer cores, fazer bons trabalhos ou saber apertar os cordões dos sapatos. Os outros dois itens referem-se à percepção que a criança tem da resposta à sua competência de realização, por parte de dois adultos significativos: a mãe e a educadora. $\mathrm{Na}$ mesma linha de leitura do anterior, parece-nos que o que é comum aos itens que saturam este factor é o facto de traduzirem a percepção de competência na realização de actividades intencionalmente ensinadas à criança, nomeadamente pela mãe e pela educadora, incluindo a percepção da avaliação que é feita por estes dois adultos. Refira-se ainda que embora a carga factorial dos itens 9 e 21 esteja abaixo do valor critério definido $(0,350)$, com base na análise de conteúdo destes itens, decidiu-se conservá-los.

\section{Fidelidade}

A fidelidade da EAPCASC foi apreciada através do cálculo do índice de consistência interna (coeficiente alfa de Cronbach). O valor de alfa de Cronbach obtido, quando se considera o total dos 31 itens foi de 0,87 . Quando se procede à mesma análise da consistência interna, considerando cada uma das sub-escalas derivadas, respectivamente, dos quatro factores identificados, obteve-se como valores de alfa de Cronbach, 0,80 para a sub-escala de percepção da qualidade da relação com a mãe; 0,82 para a sub-escala de percepção da qualidade da relação com os pares; 0,70 para a sub-escala de percepção de competência na realização de actividades naturalmente aprendidas e, finalmente, 0,64 para a subescala de percepção de competência na realização de actividades intencionalmente ensinadas. Os valores obtidos como índices de consistência interna da EAPCASC, quer a nível global, quer de cada uma das sub-escalas consideradas, são considerados satisfatórios e indicadores de certa homogeneidade entre os itens.

\section{Validade convergente discriminante}

A validade convergente discriminante $\mathrm{da}$ EAPCASC foi apreciada através do cálculo da correlação entre cada item e cada uma das sub-escalas, considerando-se como desejável que a correlação de cada item com a sub-escala de que faz parte seja superior à correlação com qualquer outra sub-escala. A Tabela 4 apresenta os valores do coeficiente de correlação de Pearson obtidos entre cada item e cada uma das quatro sub-escalas, bem como com a escala completa. 
Tabela 4 - EAPCASC: Coeficiente de correlação entre cada item e cada uma das quatro sub-escalas, e cada item e a escala total, bem como a diferença encontrada entre a correlação com a sub-escala respectiva e a correlação com a sub-escala seguinte $(\mathrm{N}=128)$

\begin{tabular}{|c|c|c|c|c|c|c|}
\hline Item & R. Mãe & R. Pares & C. Aprend. & C. Ensin. & Total & $\not \neq$ \\
\hline 1 - Sabe fazer puzzles & 0,11 & 0,01 & $0,52 *$ & 0,20 & 0,27 & 0,32 \\
\hline 2 - Tem amigos & $0,37 *$ & $0,76 *$ & 0,21 & 0,29 & $0,54 *$ & 0,39 \\
\hline 3 - Anda de balouço & 0,26 & 0,18 & $0,52 *$ & 0,29 & $0,41 *$ & 0,23 \\
\hline 4 - Mãe bem-disposta & 0,25 & $0,37 *$ & 0,19 & $0,49 *$ & $0,42^{*}$ & 0,12 \\
\hline 5 -Educadora elogia & 0,15 & 0,28 & $0,32^{*}$ & $0,55^{*}$ & $0,41 *$ & 0,23 \\
\hline 6 - Convidado ir dormir & $0,65^{*}$ & $0,40 *$ & $0,35 *$ & 0,29 & $0,58^{*}$ & 0,25 \\
\hline 7 - Trepa & $0,33^{*}$ & 0,22 & $0,69 *$ & 0,29 & $0,50^{*}$ & 0,36 \\
\hline 8 - Mãe leva a passear & $0,54 *$ & $0,31 *$ & 0,27 & $0,32 *$ & $0,49 *$ & 0,22 \\
\hline 9 - Conhece cores & 0,15 & 0,13 & 0,24 & $0,38 *$ & 0,29 & 0,18 \\
\hline 10 - Amigos p/ jogar & $0,37 *$ & $0,78 *$ & 0,29 & 0,23 & $0,55^{*}$ & 0,41 \\
\hline 11 - Aperta sapatos & 0,15 & 0,12 & 0,14 & $0,55 *$ & 0,30 & 0,40 \\
\hline 13 - Sabe contar & 0,18 & 0,26 & $0,33 *$ & $0,53 *$ & $0,41 *$ & 0,20 \\
\hline 14 - Amigos p/ brincar & 0,29 & $0,76 *$ & 0,24 & 0,29 & $0,52 *$ & 0,47 \\
\hline 15 - Consegue saltar & 0,20 & $0,32 *$ & $0,58 *$ & 0,23 & $0,43^{*}$ & 0,26 \\
\hline 16 - Mãe lê histórias & $0,62 *$ & 0,17 & 0,10 & 0,05 & $0,35^{*}$ & 0,45 \\
\hline 17 - Conhece inicial & $-0,01$ & 0,14 & 0,05 & $0,50 *$ & 0,20 & 0,36 \\
\hline 18 - Chamado $\mathrm{p} /$ brincar & $0,44^{*}$ & $0,63 *$ & $0,41 *$ & $0,40^{*}$ & $0,62 *$ & 0,19 \\
\hline 19 - Corre & 0,16 & 0,12 & $0,53 *$ & 0,18 & $0,32 *$ & 0,35 \\
\hline 20 - Mãe brinca & $0,66 *$ & $0,34 *$ & $0,43 *$ & 0,24 & $0,58^{*}$ & 0,23 \\
\hline 21 - Conhece letras & $0,37 *$ & 0,29 & 0,29 & $0,54 *$ & $0,49 *$ & 0,17 \\
\hline 22 - Convidado ir lanchar & $0,71 *$ & $0,37 *$ & $0,32 *$ & 0,28 & $0,58^{*}$ & 0,34 \\
\hline 23 - Salta pé-coxinho & 0,24 & 0,26 & $0,55 *$ & 0,23 & $0,42 *$ & 0,29 \\
\hline 24 - Mãe conversa & $0,62 *$ & $0,40^{*}$ & 0,17 & 0,25 & $0,50^{*}$ & 0,22 \\
\hline 25 - Trabalhos J. I. & 0,30 & 0,20 & $0,42 *$ & $0,52 *$ & $0,46^{*}$ & 0,10 \\
\hline 26 - Amigos emprestam & $0,41 *$ & $0,67 *$ & 0,24 & $0,33 *$ & $0,55^{*}$ & 0,26 \\
\hline 27 - Joga à bola & $0,32 *$ & 0,24 & $0,57 *$ & 0,29 & $0,47 *$ & 0,25 \\
\hline 28 - Mãe deixa ir lanchar & $0,67 *$ & $0,39 *$ & 0,30 & 0,25 & $0,56^{*}$ & 0,28 \\
\hline 29 - Sabe escrever nome & 0,22 & 0,25 & 0,17 & $0,55 *$ & $0,38^{*}$ & 0,30 \\
\hline 30 - Outros sentam junto & $0,41 *$ & $0,74 *$ & 0,19 & $0,35^{*}$ & $0,56^{*}$ & 0,33 \\
\hline 31 - É forte & $0,31 *$ & $0,32 *$ & $0,60 *$ & 0,29 & $0,49 *$ & 0,28 \\
\hline 32 - Mãe deixa dormir fora & $0,73 *$ & $0,37 *$ & 0,29 & $0,33 *$ & $0,60 *$ & 0,36 \\
\hline
\end{tabular}
${ }^{*} p<0,001$

Da leitura da Tabela 4 pode apreciar-se a validade convergente discriminante da EAPCASC, na medida em que se verifica que o coeficiente de correlação de Pearson obtido entre cada item e a sub-escala respectiva é superior ao coeficiente de correlação obtido com qualquer das outras três sub-escalas. De facto, para a quase totalidade dos itens, a diferença encontrada entre a correlação obtida com a sub-escala respectiva e a correlação obtida com a sub-escala seguinte é superior ao critério estabelecido como desejável de 0,15. Apenas dois itens são excepção, item 4 e item 25 , em que as diferenças obtidas foram respectivamente de 0,12 e
0,10. Além disso, verifica-se que os coeficientes de correlação obtidos entre cada item e a respectiva subescala são significativos $(p<0,001)$. Por outro lado, a quase totalidade dos valores de correlação (coeficiente de Pearson) obtidos entre cada item e a escala completa são também significativos $(\phi<0,001)$.

Uma vez estabelecida a estrutura da EAPCASC em quatro sub-escalas, derivadas respectivamente dos quatro factores definidos na estrutura factorial, procedeu-se ao estudo da correlação (coeficiente de Pearson) existente entre ela, e entre cada uma das sub-escalas e a escala completa. A Tabela 5 apresenta os resultados obtidos. 
Tabela 5 - EAPCASC: Coeficiente de correlação entre as quatro sub-escalas e entre cada sub-escala e a escala completa $(\mathrm{N}=128)$

\begin{tabular}{lcccc}
\hline & R. Mãe & R. Pares & C. Aprendido & C. Ensinado \\
\hline R. Mãe & & & & \\
R. Pares & $\mathbf{0 , 7 3 ^ { * }}$ & & & \\
C. Aprendido & $0,40^{*}$ & $0,36^{*}$ & & \\
C. Ensinado & $0,38^{*}$ & $0,40^{*}$ & $\mathbf{0 , 6 8 ^ { * }}$ & $0,72^{*}$ \\
Total & $0,81^{*}$ & $0,76^{*}$ & $0,72^{*}$ & \\
\hline
\end{tabular}

A Tabela 5 mostra que a correlação existente entre cada uma das sub-escalas e a escala completa é elevada, sendo significativa para $p<0,001$. Por outro lado, verifica-se que a correlação entre as duas subescalas relativas à percepção de aceitação social, isto é, a sub-escala de percepção de qualidade de relação com a mãe e a sub-escala de percepção de qualidade de relação com os pares, é maior do que a correlação encontrada entre qualquer uma destas sub-escalas e qualquer das sub-escalas de percepção de competência. Do mesmo modo, também se verifica que a correlação existente entre as duas sub-escalas de percepção de competência, isto é, a sub-escala de percepção de competência na realização de actividades naturalmente aprendidas e a sub-escala de percepção de competência na realização de actividades intencionalmente ensinadas, é superior à correlação encontrada entre qualquer uma destas sub-escalas e qualquer sub-escala de percepção de aceitação social.

\section{Medidas descritivas}

$\mathrm{Na}$ sequência do atrás referido, foram calculadas para cada criança várias notas: uma nota relativa à escala completa obtida por somatório de todos os 31 itens; uma nota relativa à Escala de Auto-Percepção de Competência Pessoal, obtida por somatório dos 17 itens de percepção de competência; uma nota relativa à Escala de Auto-Percepção de Aceitação Social, obtida por somatório dos 14 itens de percepção de aceitação social; quatro outras notas relativas a cada uma das sub-escalas definidas a partir dos quatro factores identificados: sub-escala de auto-percepção de qualidade de relação com a mãe (8 itens), sub-escala de auto-percepção de qualidade de relação com os pares (6 itens), sub-escala de auto-percepção de competência na realização de actividades naturalmente aprendidas (8 itens) e sub-escala de auto-percepção de competência na realização de actividades intencionalmente ensinadas ( 9 itens). A Tabela 6 apresenta as médias e respectivos desvios-padrão encontrados para cada uma das notas atrás referidas.

Tabela 6 - Médias e desvios-padrão obtidos na EAPCASC, pela amostra (N=128)

\begin{tabular}{llc}
\hline Escala/Sub-escala & Média & Desvio-padrão \\
\hline Escala completa & 100,43 & 12,81 \\
Auto-Percepção Competência Pessoal & 58,07 & 6,50 \\
Auto-Percep. Comp. Realiz. Activ. Aprend. & 27,54 & 3,96 \\
Auto-Percep. Comp. Realiz. Activ. Ensin. & 30,53 & 3,70 \\
Auto-Percepção Aceitação Social & 42,36 & 8,07 \\
Auto-Percep. Relação Com Mãe & 22,81 & 5,21 \\
Auto-Percep. Relação Com Pares & 19,55 & 4,01 \\
\hline
\end{tabular}

A leitura da Tabela 6 permite apreciar as medidas descritivas obtidas para a EAPCASC, junto da amostra do presente estudo. Dadas as alterações introduzidas a nível da estrutura da escala e que, obviamente, se repercutem nas medidas descritivas obtidas, não é possível comparar estes valores com resultados de outros estudos realizados.

\section{Conclusão}

Pode considerar-se que a EAPCASC portuguesa constitui um instrumento de avaliação da percepção de competência pessoal e de aceitação social junto de crianças de 5 anos com qualidades psicométricas muito satisfatórias. De facto, não só se verificou que os itens apre- sentavam um grau de sensibilidade elevado como a consistência interna da escala, bem como a sua validade convergente discriminante, uma vez assumida a estrutura resultante da análise factorial, se revelaram muito positivas.

A análise factorial conduzida permitiu a identificação de um perfil factorial diferente do proposto na escala original americana (Harter \& Pike, 1984), na medida em que emergiram quatro factores cuja análise de conteúdo dos itens que os saturam levou a denominá-los, respectivamente, percepção de qualidade de relação com a mãe, percepção de qualidade de relação com os pares, percepção de competência na realização de actividades naturalmente aprendidas e percepção de competência na realização de actividades intencionalmente ensinadas. Ou 
seja, os resultados obtidos apontam para o facto de que as crianças de cinco anos não só são capazes de discriminar entre a percepção que têm da sua competência pessoal e a percepção que têm da qualidade das relações que são capazes de estabelecer com "outros significativos", como são capazes de proceder à distinção entre domínios diferentes de competência (aprendidos e ensinados em vez de motores e cognitivos), e de diferenciar entre outros significativos (a mãe e os pares). Estes dados reforçam a perspectiva da multidimensionalidade do self e, embora as dimensões identificadas sejam outras, são compatíveis com os resultados de investigação que reconhece diferentes domínios na auto-percepção de crianças de cinco anos (Marsh, Craven \& Debus, 1998, Measelle, Ablow, Cowan \& Cowan, 1998). As análises conduzidas para determinar a fidelidade e validade convergente discriminante da EAPCASC, confirmaram a pertinência da definição de sub-escalas derivadas do padrão factorial. Os valores do coeficiente alfa de Cronbach, obtidos para a escala completa e para cada uma das quatro sub-escalas, revelaram-se satisfatórios evidenciando certa homogeneidade intra-escala. É facto que estes índices de fidelidade, no que diz respeito às duas subescalas de percepção de competência, poderiam ser significativamente melhorados se os itens que se revelaram mais fracos fossem substituídos por outros novos. Estes novos itens a incluir deveriam ser desenhados a partir da distinção estabelecida entre percepção de competência na realização de actividades naturalmente aprendidas no contexto da interacção com os pares (isto é, sem objectivos de aprendizagem intencional), e a percepção de competência na realização de actividades que implicaram num processo intencional de aprendizagem. Finalmente, notese que no que diz respeito à validade da EAPCASC, quase todos os itens atingiram o critério de validade estabelecido, permitindo que a escala seja considerada uma medida válida de avaliação da auto-percepção de competência para crianças portuguesas de idade pré-escolar.

Em suma, pode considerar-se que o estudo de adaptação da PSPCSA a uma população portuguesa de cinco anos atingiu os objectivos propostos, na medida em que a EAPCASC se revelou uma medida fiel, válida e de certo modo inovadora na avaliação da auto-percepção de competência. $\mathrm{O}$ trabalho de adaptação conduzido sobre um instrumento construído num contexto cultural diferente é, aliás, um requerimento essencial de garantia de uma utilização válida e significativa (Fantuzzo, McDermott, Manz, Hampton \& Burdick, 1996). Num momento em que a globalização está na ordem do dia, há que ter presente que as especificidades culturais continuam a existir e que é essencial que sejam tidas em conta na prática da avaliação e inter- venção psicológicas. O estudo de adaptação da PSPCSA, aqui apresentado, torna bem clara esta necessidade.

\section{Referências}

Ducharne, M. (2000). Elementos para um modelo teórico do desenvolvimento do self: os factores preditores da auto-percepção de competência em crianças de cinco anos. Porto: FPCEUP.

Fantuzzo, J. W., Mc Dermott, P. A., Manz, P. H., Hampton, V. R. \& Burdick, N. A. (1996). The Pictorial Scale of Perceived Competence and Social Acceptance: Does it work with low-income urban children? Child Development, 67(3), 1071-1084.

Fife-Schaw, C. (1995). Levels of measurement. Em G. Beackell, S. Hammond \& Fife-Schaw (Orgs.). Research methods in psychology (pp. 38-49). London: Sage Publications.

Harter, S. \& Pike, R. (1983). Procedural manual to accompany the Pictorial Scale of Perceived Competence and Social Acceptance for Young Children. Denver: University of Denver.

Harter, S. \& Pike, R. (1984). The Pictorial Scale of Perceived Competence and Social Acceptance for Young Children. Child Development, 55, 1969-1982.

Marsh, H. W., Craven, R. \& Debus, R. (1998). Structure, stability and development of young children's self-concepts: A multicohort-multiocasion study. Child Development, 69(4), 1030-1053.

Measelle, J. R., Ablow, J. C. Cowan, P. A. \& Cowan, C. P. (1998). Assessing young children's view of their academic, social and emotional lives: An evaluation of the self-perception scales of the Berkeley Puppet Interview. Child Development, 69(6), 1556-1576.

Miller, S. (1984). Experimental design and statistics. Londres: Methuen.

Norusis, M. J. (1993). SPSS Professional Statistics. Release 6.0. Chicago: SPSS Inc.

Reckase, M. (1990). Scaling techniques. Em G. Goldstein \& M. Hersen (Orgs.). Handbook of psychological assessment (pp. 41-56). New York: Pergamon Press.

Ribeiro, J. L. (1999). Investigação e avaliação em psicologia e saúde. Lisboa: Climepsi.

Recebido em novembro de 2004

Reformulado em novembro de 2004 Aprovado em dezembro de 2004

Sobre a autora:

Maria Adelina Barbosa Ducharne é psicóloga pela Universidade do Porto, doutoranda em Psicologia e professora da Faculdade de Psicologia e de Ciências da Educação da Universidade do Porto (FPCEUP), Portugal. 


\section{LabAPE \\ 政 \\ Avaliação Psicológica e \\ Educacional}

Universidade São Francisco - Programa de Pós-Graduação Stricto Sensu em Psicologia http://docente.saofrancisco.edu.br/rprimi/

e-mail labape@saofrancisco.edu.br

O LabAPE está ligado a duas das três linhas de pesquisa do Mestrado em Psicologia: Construção, validação e padronização de instrumentos de medida e Avaliação em Psicologia Educacional.

\section{Os objetivos principais são:}

1. Desenvolver e aprimorar instrumentos e técnicas de medida psicológica e educacional;

2. Investigar as aplicações práticas desses instrumentos em contextos educacionais, institucionais e organizacionais;

3. Desenvolver aplicações da Psicometria e a Teoria de Resposta ao Item;

4. Desenvolver recursos tecnológicos de informática que auxiliem a obtenção, organização, análise e interpretação de dados em psicologia e educação;

5. Organizar as informações coletadas através das pesquisas realizadas pelo grupo com o duplo propósito de (1) estabelecer parâmetros para os instrumentos pesquisados, (2) prover informações objetivas que auxiliem práticas preventivas e/ou interventoras dentro e fora da universidade.

Financiamento: Fapesp e CNPq

\section{Equipe}

Dr. Ricardo Primi (ricardo.primi@saofrancisco.edu.br)

Dra. Acácia Angeli dos Santos (acacia.santos@saofrancisco.edu.br)

Dra. Claudette Maria Medeiros Vendramini (claudette.vendramini@saofrancisco.edu.br)

Dra. Ana Paula Porto Noronha (ana.noronha@saofrancisco.edu.br) 